\title{
The syndrome of perisylvian polymicrogyria with congenital arthrogryposis
}

\author{
Annapurna Poduri ${ }^{\mathrm{a}}$, Vida Chitsazzadeh ${ }^{\mathrm{a}}$, Stefano D’Arrigo ${ }^{\mathrm{b}}$, Ermellina Fedrizzi ${ }^{\mathrm{b}}$, \\ Chiara Pantaleoni ${ }^{b}$, Daria Riva ${ }^{b}$, Claudia Busse ${ }^{c}$, Helmut Küster ${ }^{c}$, Adre Duplessis ${ }^{d}$, \\ John Gaitanis ${ }^{\mathrm{e}}$, Mustafa Sahin ${ }^{\mathrm{d}}$, Cheryl Garganta ${ }^{\mathrm{f}, 1}$, Meral Topcu ${ }^{\mathrm{g}}$, Kira A. Dies ${ }^{\mathrm{h}}$, \\ Brenda J. Barry ${ }^{i}$, Jennifer Partlow ${ }^{i}$, A. James Barkovich ${ }^{j}$, Christopher A. Walsh ${ }^{\text {h,i }}$, \\ Bernard S. Chang ${ }^{k, *}$
}

${ }^{a}$ Division of Epilepsy and Clinical Neurophysiology, Department of Neurology, Children's Hospital Boston and Harvard Medical School, Boston, MA, USA

${ }^{\mathrm{b}}$ Department of Neurodevelopmental Neurology, Fondazione IRCCS Instituto Neurologico “C. Besta”, Milan, Italy

${ }^{\mathrm{c}}$ Neonatology, University Pediatric Hospital, Greifswald, Germany

d Department of Neurology, Children's Hospital Boston and Harvard Medical School, Boston, MA, USA

e Pediatric Neurology, Hasbro Children's Hospital and Brown University School of Medicine, Providence, Rhode Island, USA

${ }^{\mathrm{f}}$ Genetics and Metabolism, Tufts Medical Center, Tufts University School of Medicine, Boston, MA, USA

${ }^{\mathrm{g}}$ Department of Child Neurology, Hacettepe University, Ankara, Turkey

h Division of Genetics, The Manton Center for Orphan Disease Research, Children's Hospital Boston and Harvard Medical School, Boston, MA, USA

${ }^{\mathrm{i}}$ Howard Hughes Medical Institute, Department of Neurology, Beth Israel Deaconess Medical Center, Boston, MA, USA

${ }^{\mathrm{j}}$ Pediatric Neuroradiology, Department of Neuroradiology, University of California, San Francisco, USA

${ }^{\mathrm{k}}$ Comprehensive Epilepsy Center, Department of Neurology, Beth Israel Deaconess Medical Center and Harvard Medical School, Boston, MA, USA

Received 28 May 2009; received in revised form 17 August 2009; accepted 18 August 2009

\begin{abstract}
Background: Bilateral perisylvian polymicrogyria (BPP) is a well-recognized malformation of cortical development commonly associated with epilepsy, cognitive impairment, and oromotor apraxia. Reports have suggested the association of BPP with arthrogryposis multiplex congenita. We sought to investigate the clinical, electrophysiological, and neuroradiological features of this combined syndrome to determine if there are unique features that distinguish BPP with arthrogryposis from BPP alone. Methods: Cases of BPP with congenital arthrogryposis were identified from a large research database of individuals with polymicrogyria. Clinical features (including oromotor function, seizures, and joint contractures), MR brain imaging, and results of neuromuscular testing were reviewed. Results: Ten cases of BPP with congenital arthrogryposis were identified. Most cases had some degree of oromotor apraxia. Only a few had seizures, but a majority of cases were still young children. Electrophysiological studies provided evidence for lower motor neuron or peripheral nervous system involvement. On brain imaging, bilateral polymicrogyria (PMG) centered along the Sylvian fissures was seen, with variable extension frontally or parietally; no other
\end{abstract}

\footnotetext{
${ }^{*}$ Corresponding author. Address: Comprehensive Epilepsy Center, E/KS-457, Department of Neurology, Beth Israel Deaconess Medical Center, Harvard Medical School, 330 Brookline Ave., Boston, MA 02215, United States. Tel.: +1 617667 2889; fax: +1 6176677919.

E-mail address: bchang@bidmc.harvard.edu (B.S. Chang).

${ }^{1}$ Formerly at Yale University School of Medicine, New Haven, CT, United States.
} 
cortical malformations were present. We did not identify obvious neuroimaging features that distinguish this syndrome from that of BPP without arthrogryposis. Conclusions: The clinical and neuroimaging features of the syndrome of BPP with congenital arthrogryposis appear similar to those seen in cases of isolated BPP without joint contractures, but electrophysiological studies often demonstrate coexistent lower motor neuron or peripheral nervous system pathology. These findings suggest that BPP with arthrogryposis may have a genetic etiology with effects at two levels of the neuraxis.

(C) 2009 Elsevier B.V. All rights reserved.

Keywords: Cortical malformation; Dysplasia; Perisylvian; Polymicrogyria; Contractures; Arthrogryposis

\section{Introduction}

Polymicrogyria, one of the most common malformations of cortical development, is characterized histologically by the appearance of an excessive number of small cortical folds, often fused together, with disordered cortical lamination [1]. Multiple region-specific forms of polymicrogyria have been described [2], but large series suggest that the perisylvian region is the most common location of polymicrogyria across the cortex [3]. Bilateral perisylvian polymicrogyria (BPP) is a syndrome characterized clinically by epilepsy, cognitive impairment, and the distinct feature of oromotor apraxia in the majority of affected cases [4]. BPP may exist as a familial condition and appears to be genetically heterogeneous $[5,6]$.

Early case series of BPP, often labeled congenital bilateral perisylvian syndrome, suggested that a minority of cases (13-33\%) were associated with arthrogryposis multiplex congenita, a condition characterized by joint contractures present from birth [4,7]. Arthrogryposis is thought to arise fundamentally from decreased fetal movement in utero, preventing normal joint development, and has multiple potential etiologies, including central or peripheral nervous system lesions, connective tissue abnormalities, maternal toxic exposures, and oligohydramnios. While the most parsimonious explanation would be that the structural brain malformation is the cause of the arthrogryposis in patients who have both BPP and arthrogryposis, it has been suggested that the presence of congenital arthrogryposis or club feet in the setting of polymicrogyria may actually reflect a separate developmental defect along the neuraxis [8-10].

Despite the recognition of congenital arthrogryposis as a clinical feature in a minority of cases of BPP, this subset of patients has not been described in detail. We sought to perform an analysis of clinical, electrophysiological, and radiological features of individuals with both BPP and congenital arthrogryposis to establish how this combined syndrome is distinct from BPP alone.

\section{Methods}

Individuals were identified by screening our large research database of individuals with cortical malforma- tions for those with diagnoses of polymicrogyria. All cases were reviewed by a pediatric neuroradiologist (A.J.B). Two investigators (A.P. and B.S.C.) analyzed the brain MRI of these cases to identify by consensus those individuals who had polymicrogyria (defined by an irregular cortical surface or irregular, scalloped gray-white junction, with increased apparent cortical thickness) that was primarily centered around the Sylvian fissure [11]. For those cases, medical records were examined to establish whether joint contractures were present at birth. Only cases for whom MRI clearly demonstrated perisylvian-predominant polymicrogyria and in whom arthrogryposis was clinically documented were included in this study. Two cases reported here, Individuals 8 and 9, were previously described by others [8] as having incomplete lissencephaly and thickened cortex on brain imaging but were reclassified as having perisylvian polymicrogyria based on the radiological criteria above [11]. This study was performed in accordance with a protocol approved by the Institutional Review Boards of Children's Hospital Boston and Beth Israel Deaconess Medical Center.

\section{Results}

\subsection{Clinical characteristics}

Ten individuals with perisylvian polymicrogyria and congenital arthrogryposis were identified from a larger group of 78 cases of perisylvian polymicrogyria (Table $1)$. Individuals ranged in age from 1 month to 19 years (mean 5.3 years); seven were female. All of the cases included in this report had evidence for arthrogryposis multiplex congenita, described as contractures present at birth in the form of club feet, flexion contractures, hyperextension of the knees, and sometimes hyperflexion of the wrists. Individual 3 was the only one with an asymmetry specifically noted in her examination, with the left knee and foot more severely affected than the right; the upper extremities were symmetrically involved. In three cases, congenital joint contractures were noted to be worse in the lower extremities than in the upper extremities. Among the seven cases for whom clinical data regarding signs of oromotor apraxia were available, six had feeding difficulty, six had drooling, and five had speech difficulties; in three cases, all of 
Table 1

Clinical characteristics of the combined syndrome of bilateral perisylvian polymicrogyria and arthrogryposis multiplex congenita.

\begin{tabular}{|c|c|c|c|c|c|c|}
\hline \multirow[t]{2}{*}{ Individual } & \multirow[t]{2}{*}{ Age/sex } & \multicolumn{3}{|l|}{ Oromotor apraxia } & \multirow[t]{2}{*}{ Congenital arthrogryposis } & \multirow[t]{2}{*}{ Seizures } \\
\hline & & Feeding difficulty & Speech difficulty & Drooling & & \\
\hline 1 & $1 \mathrm{~m} / \mathrm{M}$ & + & na & + &,$+ \mathrm{LE}>\mathrm{UE}$ & - \\
\hline 2 & $5 \mathrm{~m} / \mathrm{M}$ & + & na & + &,$+ \mathrm{LE}>\mathrm{UE}$ & - \\
\hline 3 & $11 \mathrm{~m} / \mathrm{F}$ & + & + & + & $+, \mathrm{LE}>\mathrm{UE}, \mathrm{R}>\mathrm{L}$ & - \\
\hline 4 & $2 \mathrm{y} / \mathrm{M}$ & + & + & + & + & - \\
\hline 5 & $2 \mathrm{y} / \mathrm{F}$ & + & + & + & + & - \\
\hline 6 & $3 \mathrm{y} / \mathrm{F}$ & unk & + & + & + & - \\
\hline 7 & $3 \mathrm{y} / \mathrm{F}$ & unk & unk & unk & + & + \\
\hline 8 & $6 \mathrm{y} / \mathrm{F}$ & unk & unk & unk & + & - \\
\hline 9 & $17 \mathrm{y} / \mathrm{F}$ & unk & unk & unk & + & + \\
\hline 10 & $19 \mathrm{y} / \mathrm{F}$ & + & + & unk & + & + \\
\hline
\end{tabular}

Abbreviations: na, not applicable; unk, unknown; LE, lower extremities; UE, upper extremities.

these features were present. Individual 6 was not explicitly reported to have feeding problems, but weight gain had been reportedly poor. In many cases, verbal language production was either absent or markedly delayed. Only three individuals had a history of seizures, but they were among the oldest in the series, and a majority of cases were under 5 years of age. These clinical features are similar to those that we have seen and that are reported in the literature in association with BPP without arthrogryposis.

\subsection{Neuromuscular evaluation}

For all five individuals who had nerve conduction studies (NCS)/electromyography (EMG) and/or muscle biopsy performed in the course of their diagnostic evaluations, findings from these tests were consistent with lower motor neuron or peripheral nervous system pathology (Table 2). Individual 1 had normal nerve conduction velocities and distal latencies; motor amplitudes were markedly decreased while sensory amplitudes were normal; and needle EMG revealed motor units that were decreased in number and had abnormally large amplitudes and rapid firing rates, consistent with a chronic lower motor neuron process. These findings, obtained at 5 days of age in the absence of neuroimaging data, suggested the possible diagnosis of spinal muscular atrophy at the time. Individual 4 had normal NCS/EMG; muscle biopsy showed evidence for myopathic changes on electron microscopy, with enlarged mitochondria and amplification of smooth endoplasmic reticulum. Individual 8 had NCS/EMG findings suggestive of a neurogenic process and abnormalities on muscle biopsy that also suggested a chronic neurogenic process, namely fiber type grouping and fiber type disproportion. Individual 9 had normal NCS/EMG, but muscle biopsy

Table 2

Neuromuscular evaluation in the combined syndrome of bilateral perisylvian polymicrogyria and arthrogryposis multiplex congenita.

\begin{tabular}{|c|c|c|c|c|c|}
\hline Individual & Age/Sex & Nerve conduction studies & Electromyography & Muscle biopsy & Diagnosis \\
\hline 1 & $1 \mathrm{~m} / \mathrm{M}$ & $\begin{array}{l}\text { Normal NCVs } \\
\text { Markedly decreased CMAPs, } \\
\text { normal SNAPs }\end{array}$ & $\begin{array}{l}\text { Decreased numbers of } \\
\text { motor units with abnormally } \\
\text { large amplitudes and rapid } \\
\text { firing rates }\end{array}$ & Not performed & $\begin{array}{l}\text { Chronic lower } \\
\text { motor neuron } \\
\text { process }\end{array}$ \\
\hline 4 & $2 \mathrm{y} / \mathrm{M}$ & Normal & Normal & $\begin{array}{l}\text { Myopathic changes } \\
\text { Electron microscopy: } \\
\text { enlarged mitochondria, } \\
\text { amplification of smooth } \\
\text { endoplasmic reticulum }\end{array}$ & Myopathy \\
\hline 8 & $6 y / F$ & Normal & Neurogenic findings & $\begin{array}{l}\text { Fiber type grouping and } \\
\text { fiber type disproportion }\end{array}$ & $\begin{array}{l}\text { Chronic } \\
\text { neurogenic process }\end{array}$ \\
\hline 9 & $17 \mathrm{y} / \mathrm{F}$ & Normal & Normal & $\begin{array}{l}\text { Fiber type grouping and } \\
\text { fiber type disproportion }\end{array}$ & $\begin{array}{l}\text { Chronic } \\
\text { neurogenic process }\end{array}$ \\
\hline \multirow[t]{2}{*}{10} & $19 \mathrm{y} / \mathrm{F}$ & Normal NCVs & $\begin{array}{l}\text { Changes suggesting } \\
\text { chronic denervation }\end{array}$ & Not performed & $\begin{array}{l}\text { Severe axonal motor } \\
\text { polyneuropathy or } \\
\text { distal lower motor } \\
\text { neuron process }\end{array}$ \\
\hline & & $\begin{array}{l}\text { Decreased CMAPs } \\
\text { in the distal muscles, } \\
\text { normal SNAPs }\end{array}$ & & & \\
\hline
\end{tabular}

Abbreviations: NCV, nerve conduction velocity; CMAP, compound muscle action potential; SNAP, sensory nerve action potential. 
showed evidence of a chronic neurogenic process. Neither Individual 8 nor 9 had any evidence of myopathy. Individual 10 had normal nerve conduction velocities, decreased compound muscle action potentials in the distal muscles, normal sensory nerve action potentials, and needle EMG changes suggesting chronic denervation consistent with either a severe axonal motor polyneuropathy or a distal lower motor neuron process. In both Individuals 1 and 10, an absence of fibrillation potentials and positive sharp waves was specifically noted, suggesting that the changes were consistent with a static process without evidence for ongoing denervation. Since BPP patients without arthrogryposis did not generally have a clinical indication for electrophysiological studies, a direct comparison could not be made between those with and without arthrogryposis. Nonetheless, these data suggest that BPP patients with arthrogryposis appear to share not only the cortical malformation, but also a lower motor neuron or other more distal process that is congenital and not obviously progressive.

\subsection{Radiological analysis}

Brain MR imaging was analyzed systematically in all individuals (Table 3). All cases showed clear regions of polymicrogyria, manifest as focal regions of the cortex appearing thicker than normal with some irregular festooning of the gray-white matter junction, centered around the perisylvian regions bilaterally. In addition to the clearly abnormal configuration of the cortical rib- bon, we noted an abnormal orientation of the Sylvian fissures, best seen on sagittal images, and an abnormally open appearance of the fronto-temporo-parietal opercula. Two cases, Individuals 1 and 3, had notable suprasylvian involvement that was more extensive than seen in the others. In one case, Individual 7, there was a clear posterior-anterior gradient of malformation severity, with the posterior perisylvian regions displaying more PMG than the anterior perisylvian regions.

Although the polymicrogyria was always centered along the Sylvian fissure, it frequently involved cortical regions contiguous to the perisylvian area, including more anterior or superior regions of the frontal lobe or more posterior or superior regions of the parietal lobe (Fig. 1). In three cases (Individuals 2, 5, and 10), there were separate cortical infoldings lined by polymicrogyria that did not appear immediately contiguous to the polymicrogyric perisylvian cortex. In three other cases (Individuals 6-8), the Sylvian fissure was abnormally forked along its extent, with both forked branches lined by apparent polymicrogyric cortex. In four of the ten cases (Individuals 1, 3, 4, and 10), there was significant asymmetry in the severity and extent of PMG, with the right perisylvian region more extensively involved than the left in all four. The underlying white matter appeared decreased in volume in most cases, but no other cortical malformations besides polymicrogyria were seen. These MRI findings were not distinguishable from those seen in our cases of perisylvian polymicrogyria without

Table 3

Neuroimaging findings in the combined syndrome of bilateral perisylvian polymicrogyria and arthrogryposis multiplex congenita.

\begin{tabular}{|c|c|c|c|c|c|c|}
\hline Individual & Age/sex & Symmetric & $\begin{array}{l}\text { Pattern if } \\
\text { asymmetric }\end{array}$ & $\begin{array}{l}\text { Distribution of } \\
\text { predominant PMG }\end{array}$ & Additional extent of PMG & Other abnormalities \\
\hline 1 & $1 \mathrm{~m} / \mathrm{M}$ & - & $\mathrm{R}>\mathrm{L}$ & $\begin{array}{l}\text { Suprasylvian } \\
\text { Posterior frontal }\end{array}$ & Right insula & None \\
\hline 2 & $5 \mathrm{~m} / \mathrm{M}$ & + & - & Perisylvian & $\begin{array}{l}\text { Bilateral medial } \\
\text { parieto-occipital } \\
\text { infoldings }\end{array}$ & $\begin{array}{l}\text { Thin } \\
\text { corpus } \\
\text { callosum }\end{array}$ \\
\hline 3 & $11 \mathrm{~m} / \mathrm{F}$ & - & $\mathrm{R}>\mathrm{L}$ & $\begin{array}{l}\text { Perisylvian } \\
\text { Suprasylvian }\end{array}$ & None & None \\
\hline 4 & $2 \mathrm{y} / \mathrm{M}$ & - & $\mathrm{R}>\mathrm{L}$ & Perisylvian & Bilateral parietal & $\begin{array}{l}\text { Small splenium } \\
\text { of corpus callosum } \\
\text { Large ventricles }\end{array}$ \\
\hline 5 & $2 \mathrm{y} / \mathrm{F}$ & + & - & Perisylvian & $\begin{array}{l}\text { Bilateral parietal } \\
\text { separate infoldings }\end{array}$ & None \\
\hline 6 & $3 \mathrm{y} / \mathrm{F}$ & + & - & Perisylvian & $\begin{array}{l}\text { Bilateral superolateral } \\
\text { frontal, forked from SF }\end{array}$ & None \\
\hline 7 & $3 \mathrm{y} / \mathrm{F}$ & + & - & $\begin{array}{l}\text { Perisylvian } \\
(\mathrm{P}>\mathrm{A})\end{array}$ & Left parietal, forked from SF & None \\
\hline 8 & $6 \mathrm{y} / \mathrm{F}$ & + & - & Perisylvian & Left parietal, forked from SF & $\begin{array}{l}\text { Mild atrophy } \\
\text { of cerebellar vermis }\end{array}$ \\
\hline 9 & $17 \mathrm{y} / \mathrm{F}$ & + & - & Perisylvian & None & None \\
\hline 10 & $19 \mathrm{y} / \mathrm{F}$ & - & $\mathrm{R}>\mathrm{L}$ & Perisylvian & $\begin{array}{l}\text { Bilateral superolateral } \\
\text { frontal and parietal/ } \\
\text { posterior temporal }\end{array}$ & None \\
\hline
\end{tabular}

Abbreviations: PMG, polymicrogyria; R, right; L, left; P, posterior; A, anterior; SF, Sylvian fissure. 

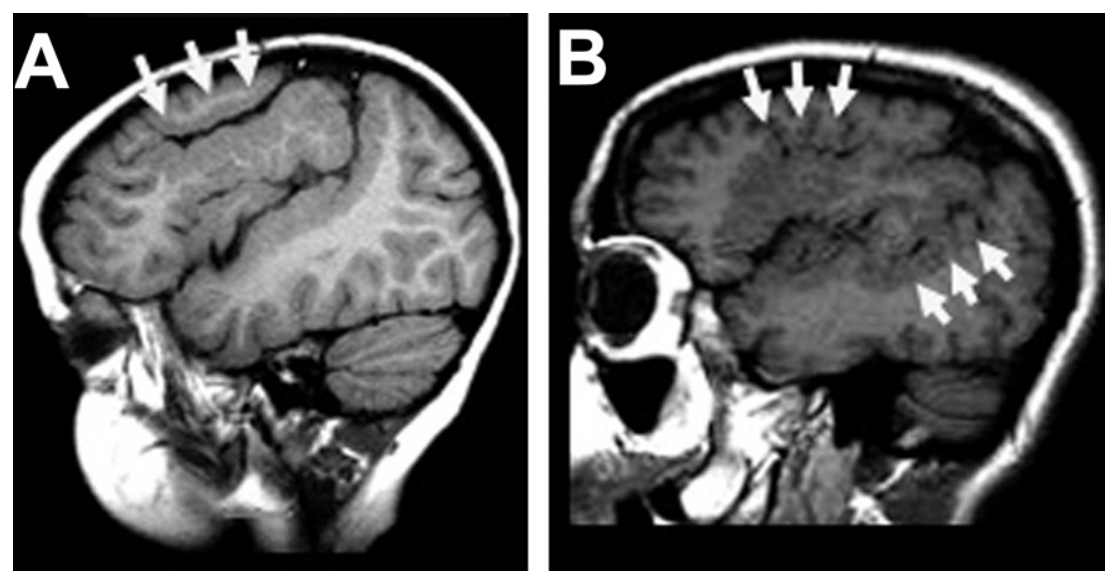

Fig. 1. Brain MR imaging of cases with bilateral perisylvian polymicrogyria and arthrogryposis multiplex congenita. T1-weighted sagittal images show examples of the MR appearance of bilateral perisylvian polymicrogyria in individuals with congenital arthrogryposis. In Individual 6 (A), there is a forked appearance to the Sylvian fissure at the vertex, with a superolateral sulcus lined by polymicrogyria (white arrows) in addition to the perisylvian abnormality. In Individual 10 (B), there is a similar appearance of perisylvian and superolateral frontal polymicrogyria, but in addition a separate cleft beginning in the parietal region and extending into the posterior superior temporal lobe is lined by polymicrogyria as well (white arrows).

arthrogryposis or from those reported for the isolated BPP syndrome in the literature.

Only three individuals had had imaging of the spinal cord performed as part of their clinical evaluation: Individuals 3 and 9 had had normal MRI of the spinal cord, and Individual 8 had diffuse atrophy of the spinal cord [8].

\section{Discussion}

Here we describe in detail the features of ten cases with both BPP and arthrogryposis multiplex congenita. Our findings demonstrate that the combined syndrome of BPP with congenital arthrogryposis shares the typical clinical and neuroradiological features characteristic of BPP more generally, but electrophysiological studies suggest a lower motor neuron or peripheral nervous system etiology for the joint contractures. For example, the clinical presentation of most cases of BPP without arthrogryposis includes oromotor apraxia [4,7]; indeed, our cases with arthrogryposis also have a high prevalence of oromotor apraxia. Although epilepsy is present in only $30 \%$ of our cases, compared to up to $87 \%$ in other BPP series [4], our cases were quite young. Seizures had developed in the majority of our cases older than 5 years. Radiological analysis does not reveal any distinct characteristics of the polymicrogyria that might distinguish this group from others with BPP. Specifically, in our comparison of the MR imaging of BPP cases with and without arthrogryposis, we found no obvious differences in the location, pattern, or extent of polymicrogyria, nor were there other major cortical malformations seen in the group with arthrogryposis.

Our findings, in the subset of cases with electrophysiological data available, support the idea of a lower motor neuron or peripheral nervous system etiology of decreased fetal joint mobility in these cases with an identified central nervous system malformation. If there is a cortically based cause for arthrogryposis in some or all of these individuals, it does not appear to lead overtly to any other clinical or imaging features that distinguish them from individuals with typical BPP without joint contractures.

Multiple hypotheses have been proposed to explain the presence of congenital joint contractures in some patients with polymicrogyria; these include an in utero vascular insult affecting both central and peripheral nervous systems, a common developmental mechanism of altered migration in both the brain and spinal cord, and a direct central effect of the brain malformation on fetal joint mobility $[8,9,12,13]$. Based on our neurophysiological observations, we hypothesize that the coexistence of BPP and arthrogryposis in a combined clinical syndrome may also be the result of an underlying genetic defect that is evident at several levels of the nervous system, not just in the cortex alone. Other investigators have shown imaging evidence of spinal cord atrophy (including in one case in this series) or electrophysiological evidence of anterior horn cell dysfunction, also suggesting that the brain malformation may not be directly responsible for the fetal motor difficulties $[8,10]$. Interestingly, the four individuals with asymmetry of the PMG on MRI had symmetric clinical examination findings, and the one individual with an asymmetric examination did not have an MRI asymmetry. These combined observations further suggest that the basis for the arthrogryposis seen in conjunction with BPP may not be due to the brain malformation.

Our work has several limitations. First, this is a relatively small number of cases, although ten cases appear to be the largest documented series of BPP with congen- 
ital arthrogryposis and our five cases with EMG data are the largest group with such evidence. A likely explanation for the paucity of EMG data and spinal MRI in cases of BPP and arthrogryposis is that clinicians treating children with this condition may attribute the entire clinical presentation to the brain malformation and not pursue further evaluation to localize the lesion responsible for the arthrogryposis. Second, due to the nature of polymicrogyria and its variable anatomic extent from case to case, we could only qualitatively assess the location and pattern of the malformation in each individual in order to determine its comparability to our other cases and to those described in the literature. In addition, the wide range of ages among our cases leaves some clinical uncertainty as to what the true frequency of epilepsy will be as the younger children grow older. Furthermore, the oromotor apraxia makes accurate evaluation of cognitive function difficult in the youngest children.

Nevertheless, our findings have both neurobiological and clinical significance. From a developmental point of view, they are consistent with the idea that congenital arthrogryposis in the presence of cerebral malformations may arise from a lower motor neuron or peripheral nervous system cause. Precisely how the second lesion outside the cortex is related to the brain malformation is not understood in detail, but we strongly suspect that this constellation of findings stems from a genetic abnormality in some cases, giving rise to defects at multiple levels of the nervous system. For clinicians caring for patients with these disorders, our findings serve as further examples of the phenotypic heterogeneity that can occur with cortical malformations, even when patients share a radiologically similar pattern of region-specific polymicrogyria on brain imaging. That the localization of the defect giving rise to arthrogryposis can apparently range within the nervous system from spinal cord (predominantly lower motor neuron) to muscle suggests that this condition may be genetically heterogeneous. Future studies with more subjects, perhaps employing quantitative neuroimaging analysis techniques, might allow us to understand even better the brain structure-function relationships in BPP as well as other malformations of cortical development. With the advent of less invasive electrodiagnostic techniques, we expect that more patients with BPP and arthrogryposis will ultimately have detailed characterization of the localization underlying their joint contractures.

\section{Disclosure of conflicts of interest}

None of the authors has any conflict of interest to disclose.

\section{Acknowledgments}

We thank those patients and families who have participated in our studies on cortical malformations. A.P. was supported by the Eleanor \& Miles Shore Scholar Award from Harvard Medical School. B.S.C. (Grant K23NS049159), A.J.B., and C.A.W. (Grant R37NS35129) were supported by the NIH/NINDS. C.A.W. is an Investigator of the Howard Hughes Medical Institute.

\section{References}

[1] Harding B, Copp A. Malformations. In: Graham DILP, editor. Greenfield's neuropathology. London: Arnold; 1997. p. 387-533.

[2] Chang BS, Piao X, Giannini C, et al. Bilateral generalized polymicrogyria (BGP): a distinct syndrome of cortical malformation. Neurology 2004;62:1722-8.

[3] Barkovich AJ, Hevner R, Guerrini R. Syndromes of bilateral symmetrical polymicrogyria. AJNR Am J Neuroradiol 1999;20:1814-21.

[4] Kuzniecky R, Andermann F, Guerrini R. Congenital bilateral perisylvian syndrome: study of 31 patients. The CBPS Multicenter Collaborative Study. Lancet 1993;341:608-12.

[5] Guerreiro MM, Andermann E, Guerrini R, et al. Familial perisylvian polymicrogyria: a new familial syndrome of cortical maldevelopment. Ann Neurol 2000;48:39-48.

[6] Villard L, Nguyen K, Cardoso C, et al. A locus for bilateral perisylvian polymicrogyria maps to Xq28. Am J Hum Genet 2002;70:1003-8.

[7] Gropman AL, Barkovich AJ, Vezina LG, Conry JA, Dubovsky EC, Packer RJ. Pediatric congenital bilateral perisylvian syndrome: clinical and MRI features in 12 patients. Neuropediatrics 1997;28:198-203.

[8] Fedrizzi E, Botteon G, Inverno M, Ciceri E, D'Incerti L, Dworzak F. Neurogenic arthrogryposis multiplex congenita: clinical and MRI findings. Pediatr Neurol 1993;9:343-8.

[9] Kammoun F, Tanguy A, Boesplug-Tanguy O, Bensahel H, Khouri N, Landrieu P. Club feet with congenital perisylvian polymicrogyria possibly due to bifocal ischemic damage of the neuraxis in utero. Am J Med Genet A 2004;126A:191-6.

[10] Clark M, Pitt M, Neville BG. Lower motor neuron involvement in perisylvian polymicrogyria. Dev Med Child Neurol 2006;48:842-6.

[11] Raybaud CGN Canto-Moreira N, Poncet M. High-definition magnetic resonance imaging identification of cortical dysplasias: micropolygyria versus lissencephaly. In: Guerrini R, Carrozzo R, Zifkin BG, et al., editors. Dysplasias of cerebral cortex and epilepsy. Philadelphia: Lippincott-Raven; 1996. p. 131-43.

[12] Banker B. Congenital deformities. In: Engel AG, FranziniArmstrong C, editors. Myology. New York: McGraw-Hill; 2004. p. 1931-62.

[13] Hageman G, Willemse J, van Ketel BA, Verdonck AF. The pathogenesis of fetal hypokinesia. A neurological study of 75 cases of congenital contractures with emphasis on cerebral lesions. Neuropediatrics 1987;18:22-33. 